\title{
Lymphoepithelial Carcinoma of the Salivary Gland: Morphologic Patterns and Imaging Features on CT and MRI
}

\author{
X. Ban, J. Wu, Y. Mo, Q. Yang, X. Liu, C. Xie, and R. Zhang
}

\begin{abstract}
BACKGROUND AND PURPOSE: Lymphoepithelial carcinoma is a rare salivary gland lesion. We retrospectively reviewed CT and MR imaging features of salivary gland lymphoepithelial carcinoma to determine their imaging features and morphologic patterns.
\end{abstract}

MATERIALS AND METHODS: The clinical data, CT, and MR imaging findings of 28 patients with histologically proved lymphoepithelial carcinoma of the salivary gland were retrospectively reviewed. Morphologic patterns of the lesions were categorized into 3 types on the basis of margin and shape.

RESULTS: There were 17 men and 11 women with a mean age of 39.3 years; $96.4 \%$ of patients were positive for Epstein-Barr virus both on histologic staining and Epstein-Barr virus serology. Tumors were parotid in 18 patients, submandibular in 8 patients, sublingual in 1 patient, and palatal in 1 patient. Most tumors (57.1\%) manifested as a partially or ill-defined mass with a lobulated or plaque-like shape. Homogeneous enhancement was found in 16 patients, while heterogeneous enhancement was found in 12, including 4 patients with intratumoral necrosis. Invasion into adjacent structures was found in 5 patients; $60.7 \%$ of patients exhibited abnormal lymph nodes, with nodal necrosis in 3 patients.

CONCLUSIONS: The characteristic lobulated or plaque-like shape, with a partially or ill-defined margin, of a salivary gland mass associated with ipsilateral lymphadenopathy may suggest a preoperative diagnosis of lymphoepithelial carcinoma.

ABBREVIATIONS: EBER $=$ Epstein-Barr virus-encoded small RNA; LEC $=$ lymphoepithelial carcinoma

ymphoepithelial carcinoma (LEC) is an uncommon malignant neoplasm characterized by undifferentiated malignant epithelial cells with marked infiltration of lymphoid cells in the stroma. ${ }^{1,2}$ LEC most frequently occurs in the nasopharynx, but it also has been reported arising in various organs such as salivary glands, lungs, thymus, stomach, larynx, soft palate, uterus, bladder, and skin. ${ }^{1,3-5}$ LEC of the salivary gland, first described by Hilderman et al in $1962,{ }^{6}$ is very rare and accounts for $0.3 \% \sim 5.9 \%$ of malignant tumors of the salivary gland; it also has a striking geographic and ethnic distribution. ${ }^{1,7,8}$ Most LECs of the salivary glands have been reported in Inuit-Yupik and Chinese

Received December 23, 2013; accepted after revision February 11, 2014

From the Medical Imaging and Minimally Invasive Interventional Center and State Key Laboratory of Oncology in Southern China, Cancer Center, Sun Yat-sen University, Guangzhou, China.

X. Ban and J. Wu contributed equally to this work.

All authors have no conflict of interest.

Please address correspondence to Rong Zhang, MD, No 651 Dongfeng Rd East, Medical Imaging and Minimally Invasive Interventional Center and State Key Laboratory of Oncology in Southern China, Cancer Center, Sun Yat-Sen University, Guangzhou, 510060, China; e-mail: zhangr@sysucc.org.cn

http://dx.doi.org/10.3174/ajnr.A3940 populations, with a strong association with the Epstein-Barr virus. ${ }^{5}$ The clinical and pathologic features, treatment, and prognosis of LEC of the salivary gland have been extensively reported. ${ }^{1,2,5,9}$ Limited literature exists, however, describing the imaging features of LEC of the salivary gland.

We retrospectively analyzed CT and MR imaging features in a series of 28 patients with pathologically proved salivary gland LEC to identify any distinct morphologic patterns and imaging features that could be useful for their diagnosis and differential diagnosis.

\section{MATERIALS AND METHODS \\ Patients}

Twenty-eight patients with pathologically confirmed LEC of a major or minor salivary gland were enrolled retrospectively between February 2001 and June 2012. This study was approved by our institutional review board, and patient informed consent was not required in accordance with the requirements of a retrospective study. Clinical data, including age, sex, clinical presentation, laboratory examinations, treatments, and outcomes, were reviewed. 

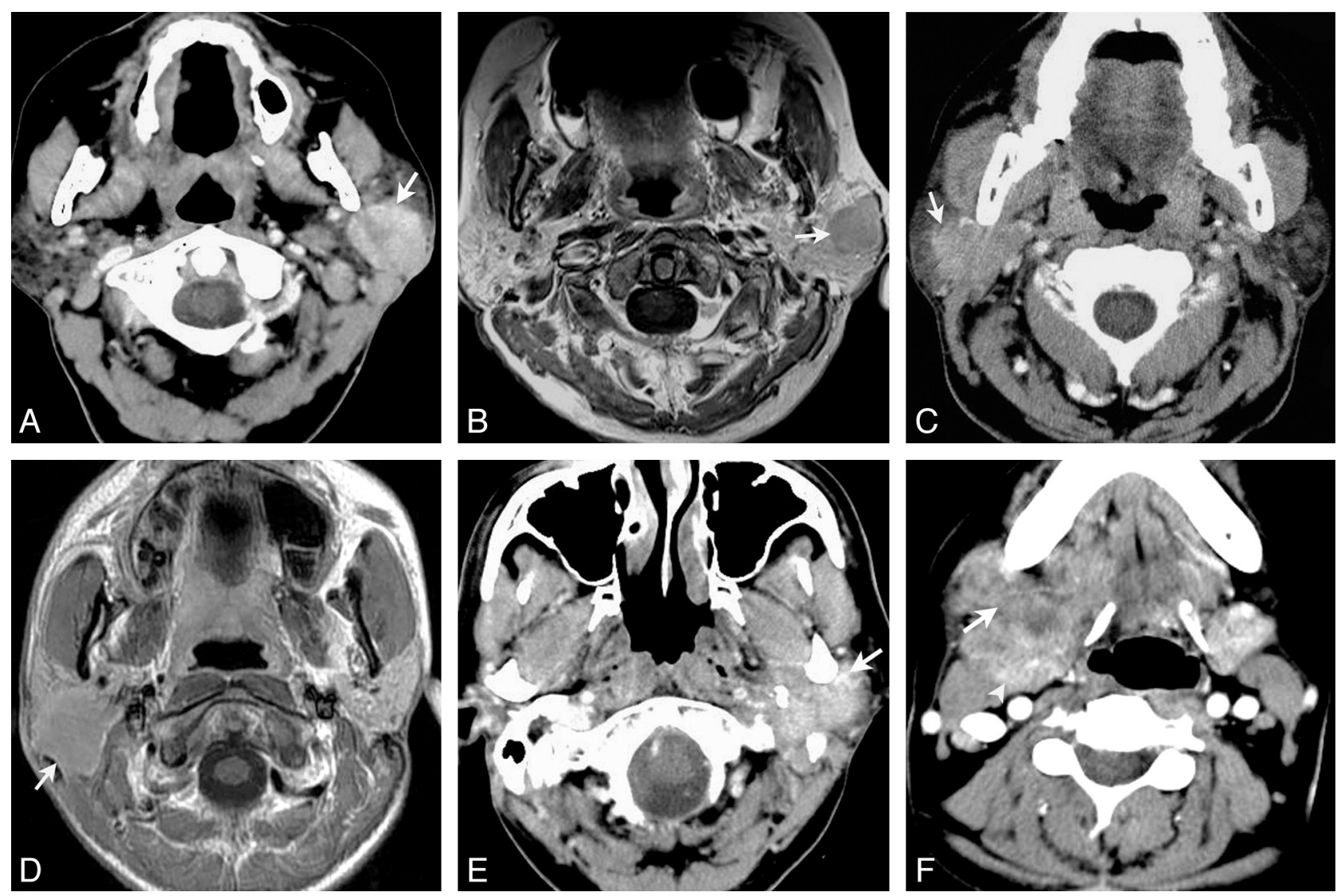

FIG 1. Morphologic patterns of salivary gland LECs. Type 1: Axial contrast-enhanced CT $(A)$ and axial gadolinium-enhanced T1-weighted imaging (B) show round masses with well-defined margins located in the left parotid glands (arrows). Type 2: Axial contrast-enhanced CT (C) and axial gadolinium-enhanced T1-weighted imaging $(D)$ show masses with plaque-like $(C)$ and lobulated $(D)$ shapes and partially defined margins located in the right parotid glands (arrows). Type 3: Axial contrast-enhanced CT shows masses with irregular shapes, ill-defined margins, and diffuse invasive growth (arrow) located in the left parotid $(E)$ and right submandibular $(F)$ glands, respectively. Normal submandibular gland tissue is displaced posteriorly and medially (arrowhead).

\section{Imaging Techniques}

All patients had cross-sectional imaging before therapy. Of 28 patients, 22 had CT examinations, 5 had MR imaging, and the remaining patient had both $\mathrm{CT}$ and MR imaging. Unenhanced and contrast-enhanced CT and MR imaging were performed in all patients.

CT was performed by using either 16-section CT (Brilliance TM16; Philips Healthcare, Best, the Netherlands) with an axial collimation of $16 \times 0.75 \mathrm{~mm}$ and a 1.2 pitch $(n=17)$ or by using dual-section CT (Twin FLASH; Philips Heathcare) with an axial collimation of $2 \times 1 \mathrm{~mm}$ and a 1.0 pitch $(n=6)$. Other parameters included the following: section thickness/gap, $3 / 3 \mathrm{~mm}(n=$ 17) or $5 / 5 \mathrm{~mm}(n=6)$; current, $250 \mathrm{~mA}$; and voltage, $125 \mathrm{kV}$. Contrast-enhanced CT was performed after intravenous injection of iopromide at a dosage of $1.5 \mathrm{~mL} / \mathrm{kg}$ of body weight (Ultravist 300; Schering, Berlin, Germany).

MR imaging was performed on a $1.5 \mathrm{~T}$ system (Signa Horizon LX, HighSpeed; GE Healthcare, Milwaukee, Wisconsin). The sequences included fast spin-echo T2-weighted images $(\mathrm{TR} / \mathrm{TE}=2000 / 120 \mathrm{~ms})$ in the axial plane; and spin-echo T1weighted images ( $\mathrm{TR} / \mathrm{TE}=450 / 15 \mathrm{~ms}$ ) in the axial, coronal, and sagittal planes. Others parameters included a matrix of $256 \times 256$, FOV of $300 \sim 380 \mathrm{~mm}$, and a section thickness/gap of $5 / 0.5 \mathrm{~mm}$ for the axial plane and $4 / 0.4 \mathrm{~mm}$ for the coronal

\footnotetext{
1814 Ban Sep 2014 www.ajnr.org
}

and sagittal planes. Contrast-enhanced sagittal and transverse T1-weighted images and contrast-enhanced coronal T1weighted images with fat suppression were obtained after intravenous injection of gadopentetate dimeglumine (Magnevist; Schering, Berlin, Germany) at a dosage of $0.2 \mathrm{mmol} / \mathrm{kg}$ of body weight. The main parameters were the same as those used preinjection.

\section{Imaging Analysis}

All images were reviewed by 2 experienced radiologists (Y.M. and C.X.) by consensus. Tumors were evaluated with regard to the location, size, margin, shape, attenuation or signal intensity (compared with adjacent muscle on CT or T1WI and compared with the contralateral normal salivary gland on T2WI), lesion texture (homogeneous or heterogeneous; with or without calcification, cystic areas, and necrosis), pattern and degree of contrast enhancement, and involvement of adjacent structures. Tumor size was measured in maximal dimensions on the transverse plane. The margin of the lesion was considered welldefined if more than two-thirds of the margin was sharply demarcated from the surrounding tissue and ill-defined if less than one-third of the margin was sharply defined, while intermediate cases were considered partially defined. The shape of 
Table 1: Clinical profiles of 28 patients with LEC of the salivary gland

\begin{tabular}{lc}
\hline \multicolumn{1}{c}{ Characteristics } & $\begin{array}{c}\text { No. of Patients/ } \\
\text { Values }\end{array}$ \\
\hline Sex & \\
Female & $11(39.3 \%)$ \\
Male & $17(60.7 \%)$ \\
Age (mean) (yr) & $12 \sim 60(39.3)$ \\
$<20$ & $4(14.3 \%)$ \\
$\geq 20 \sim<30$ & $4(14.3 \%)$ \\
$\geq 30 \sim<40$ & $6(21.4 \%)$ \\
$\geq 40 \sim<50$ & $4(14.3 \%)$ \\
$\geq 50 \sim<60$ & $8(28.6 \%)$ \\
$\geq 60$ & $2(7.1 \%)$ \\
Symptom duration (mean) (mo) & $0 . \sim 120(25)$ \\
$<24$ & $16(57.1 \%)$ \\
$\geq 24$ & $12(42.9 \%)$ \\
Epstein-Barr virus & \\
Positive & $27(96.4 \%)$ \\
Negative & $1(3.6 \%)$ \\
Clinical staging & \\
I & $1(3.6 \%)$ \\
II & $7(25.0 \%)$ \\
III & $8(28.5 \%)$ \\
IV & $12(42.9 \%)$ \\
Treatment & \\
Surgery & $7(25 \%)$ \\
Surgery and radiotherapy & $18(64.2 \%)$ \\
Surgery, radiotherapy, and chemotherapy & $2(7.2 \%)$ \\
Radiotherapy and chemotherapy & $1(3.6 \%)$ \\
Local recurrence & $3(10.7 \%)$ \\
Distant metastasis & $4(14.3 \%)$ \\
\hline &
\end{tabular}

the lesion was classified as round/oval, lobulated, and/or irregular. Patterns of enhancement were categorized as homogeneous or heterogeneous. Cystic areas were defined as having relatively homogeneous low attenuation $(\leq 20 \mathrm{HU})$ without enhancement and with a relatively clear boundary, while necrotic areas were defined as having heterogeneous low attenuation without enhancement and with an unclear boundary. The degree of enhancement was graded as poor, moderate, and intense, with "poor" defined as attenuation or signal intensity lower than or similar to that of the adjacent muscle, "moderate" as greater than that of the muscle but lower than or similar to that of the contralateral normal submandibular gland, and "intense" as greater than that of the contralateral normal submandibular gland.

The morphologic patterns of tumors were categorized into 3 types according to the margin and shape of the lesions: In type 1 , masses were round/ovoid with a well-defined margin (Fig 1A, $-B$ ); in type 2, masses showed a lobulated or plaque-like shape with a partially or ill-defined margin (Fig $1 C,-D$ ); and in type 3, masses showed an irregular shape with an ill-defined margin and diffuse invasive growth (Fig $1 E,-F)$.

The size, distribution, and attenuation or signal-intensity features of cervical lymphadenopathy were also evaluated. Cervical nodes were subdivided according to the specific anatomic subsites involved, and the assignment of level was predicated on imagingbased delineation of the cervical lymph nodes. ${ }^{10}$ The diagnosis of node involvement was defined on the basis of central necrosis and size criteria. ${ }^{11}$
Table 2: Imaging characteristics of 28 patients with LEC of the salivary gland

\begin{tabular}{|c|c|}
\hline Characteristics & $\begin{array}{c}\text { No. of Patients/ } \\
\text { Values }\end{array}$ \\
\hline \multicolumn{2}{|l|}{ Tumor location } \\
\hline Parotid & $18(64.3 \%)$ \\
\hline Submandibular gland & $8(28.5 \%)$ \\
\hline Palate & $1(3.6 \%)$ \\
\hline Sublingual gland & $1(3.6 \%)$ \\
\hline Tumor size $(\mathrm{cm})$ & $1.6 \sim 7(3.5)$ \\
\hline \multicolumn{2}{|l|}{ Tumor margin } \\
\hline Partially defined & $13(46.4 \%)$ \\
\hline Well-defined & $5(17.9 \%)$ \\
\hline Ill-defined & $10(35.7 \%)$ \\
\hline \multicolumn{2}{|l|}{ Morphologic patterns } \\
\hline Type 1 & $5(17.9 \%)$ \\
\hline Type 2 & $16(57.1 \%)$ \\
\hline Type 3 & $7(25.0 \%)$ \\
\hline \multicolumn{2}{|l|}{ Inner nature } \\
\hline Necrosis & $4(14.3 \%)$ \\
\hline Calcification & $0(0 \%)$ \\
\hline \multicolumn{2}{|c|}{ Density on unenhanced CT $(n=23)$} \\
\hline Slightly hypodense & $19(82.6 \%)$ \\
\hline Isodensity & $4(17.4 \%)$ \\
\hline \multicolumn{2}{|c|}{ Enhancement degree on CT ( $n=23)$} \\
\hline Poor & $6(26.1 \%)$ \\
\hline Moderate & $14(56.5 \%)$ \\
\hline Intense & $3(17.4 \%)$ \\
\hline \multicolumn{2}{|c|}{ Signal intensity on MR imaging $(n=6)$} \\
\hline \multicolumn{2}{|c|}{ TIWI } \\
\hline Hyperintense & $1(16.7 \%)$ \\
\hline Isointense & $5(83.3 \%)$ \\
\hline \multicolumn{2}{|l|}{$\mathrm{T} 2 \mathrm{WI}$} \\
\hline Hypointense & $4(66.7 \%)$ \\
\hline Hyperintense & $2(33.3 \%)$ \\
\hline \multicolumn{2}{|c|}{ Enhancement degree on MR imaging $(n=6)$} \\
\hline Poor & $1(16.7 \%)$ \\
\hline Moderate & $4(66.6 \%)$ \\
\hline Intense & $1(16.7 \%)$ \\
\hline \multicolumn{2}{|c|}{ Inner nature after contrast enhancement } \\
\hline Homogeneous & $16(57.1 \%)$ \\
\hline Heterogeneous & $12(42.9 \%)$ \\
\hline Adjacent structure invasion & $5(17.9 \%)$ \\
\hline Bone destroyed & $2(7.1 \%)$ \\
\hline Pathologic lymph nodes & $17(60.7 \%)$ \\
\hline
\end{tabular}

\section{RESULTS}

\section{Clinical Features}

The clinical profiles of 28 patients with LEC of the salivary gland are summarized in Table 1. All patients were southern Chinese, with 17 males and 11 females, 12-60 years of age, with a mean of 39.3 years. Of 28 patients, 20 presented with a slowly growing, palpable mass and 8 experienced a recent, rapidly growing mass. Four patients (3 with rapidly growing tumors and 1 with a slowly growing tumor) had pain or tenderness. One patient with a rapidly growing tumor had progressive facial palsy. The duration of symptoms ranged from 0.2 months to 10 years (mean, 25 months), with $42.9 \%$ (12/28) of patients having symptoms for $>2$ years. Tumors were parotid in 18 patients, submandibular in 8 patients, sublingual in 1 patient, and arising from the upper palate in 1 patient. One patient had a history of non-Hodgkin lymphoma 8 years prior. Twenty-seven cases were positive for Epstein-Barr virus on EBER (Epstein-Barr virus-encoded small RNA) in situ hybridization. The possibility of a metastasis from primary nasopharyngeal carcinoma was excluded in all cases by di- 

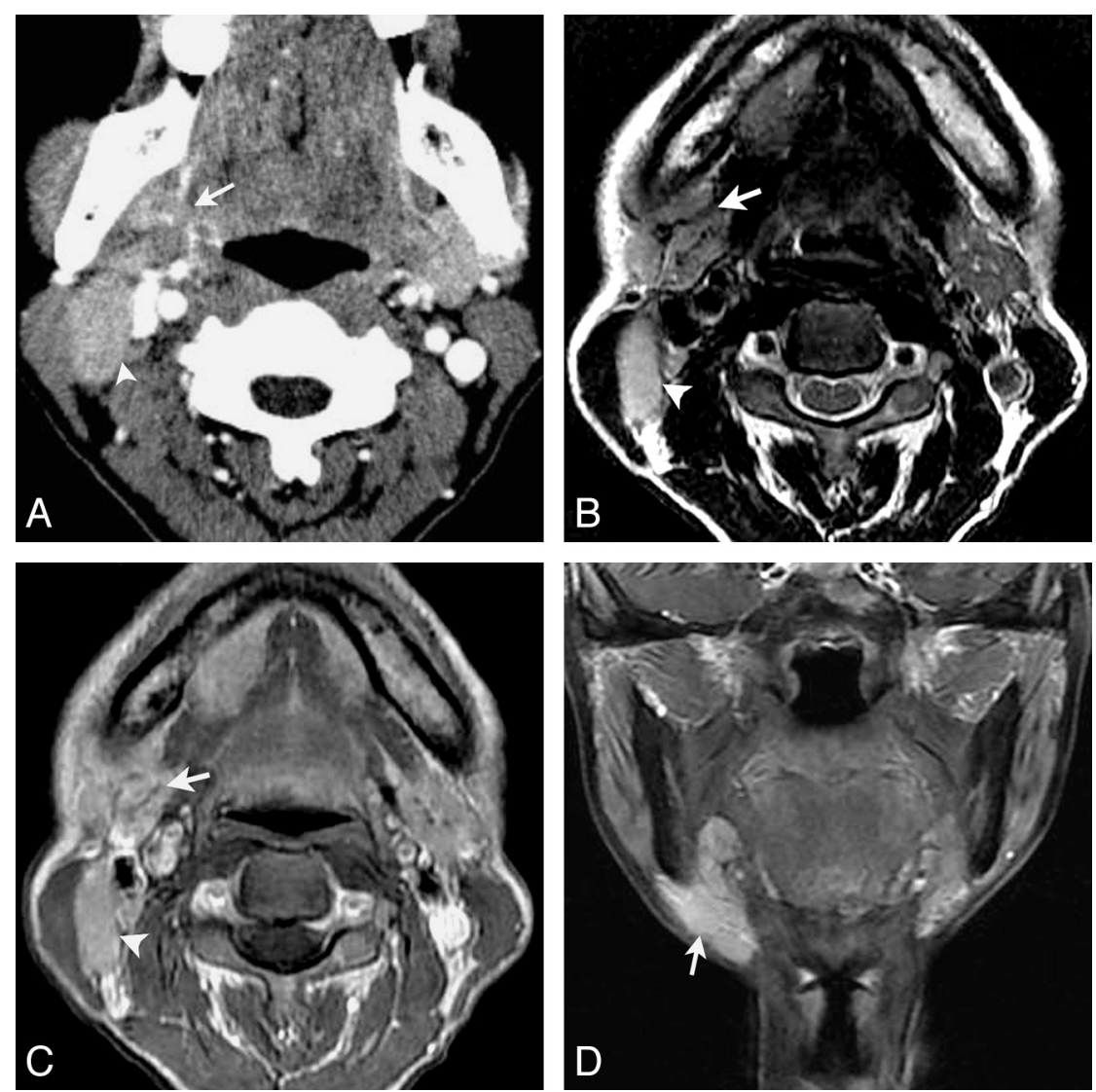

FIG 2. LEC of the right submandibular gland in a 53-year-old woman with a painless mass in the right neck for 1 year. Axial contrast-enhanced CT $(A)$ shows an ill-defined mass in the right submandibular gland with heterogeneous moderate enhancement (arrow). An enlarged lymph node in level Ila is also noted and shows a homogeneous enhancement (arrowhead). Axial T2-weighted image $(B)$ shows a heterogeneous slight hyperintensity mass with curvilinear and linear hypointensity within it (arrow) and an enlarged lymph node in level lla (arrowhead). Gadolinium-enhanced axial T1-weighted image $(C)$ and coronal T1-weighted image with fat saturation $(D)$ show the mass with a heterogeneous enhancement with hypoenhanced strands within it. An enlarged region of the lymph node in level lla is also noted and shows homogeneous enhancement (arrowhead).

rect examination $(n=28)$ and biopsy of the nasopharynx $(n=8)$ by using fiberoptic nasopharyngoscopy. Of our patients, $42.9 \%$ were classified as having stage IV, followed by stage III (28.5\%), stage II $(25.0 \%)$, and stage I (3.6\%).

Preoperative fine-needle aspiration cytology was performed in 7 patients. Two patients were correctly diagnosed as having LEC, 1 patient was suspicious for having a lymphoepithelial lesion with malignancy, 1 patient was diagnosed as having benign reactive lymphoproliferation, and the other 3 patients were diagnosed as having metastatic poorly differentiated squamous carcinoma. Twenty-seven of 28 patients underwent surgical resection to confirm the definitive diagnosis. After surgery, 18 of 27 patients received postoperative radiation therapy to the gland area and the ipsilateral upper neck with a dose range from 50 to $65 \mathrm{~Gy}$, while 2 patients received postoperative radiation therapy $(50 \mathrm{~Gy})$ and chemotherapy. Only 1 patient underwent radiation therapy (50 Gy) and chemotherapy without surgery.

Patient follow-up ranged from 5 to 123 months (mean, 41 months). Local recurrence was found in 3 patients from 12 to 28 months after surgery. Four patients, including 2 patients with local recurrence, developed distant metastases to the brain $(n=$
$1)$, lung $(n=1)$, and liver $(n=2)$ from 7 to 77 months after surgery.

\section{Imaging Findings}

The imaging characteristics of 28 patients are summarized in Table 2. Twenty-eight primary tumor masses were found in 28 patients. Lesions were solitary and unilateral in all 28 patients. The parotid gland was the most frequent location (18 of 28 patients, $64.3 \%$ ) with lesions in the superficial lobe in 11 patients, in the deep lobe in 1 patient, and in the superficial and deep lobes in 6 patients. Other less commonly involved locations were the submandibular gland $(28.5 \%, n=8)$ (Fig 2), the sublingual gland $(3.6 \%, n=1)$ (Fig 3$)$, and the palate $(3.6 \%, n=1)(\mathrm{Fig} 4)$. The size of the lesions ranged from 1.6 to 7.0 $\mathrm{cm}$, with a mean of $3.5 \mathrm{~cm}$.

The margin was partially defined in 13 patients $(46.4 \%)$, ill-defined in 10 patients (35.7\%), and well-defined in 5 patients (17.9\%). Of the 13 partially defined masses, 7 were lobulated, 3 were plaquelike, and 3 were irregular. Of the 10 illdefined masses, 5 were lobulated, 1 was plaque-like, and 4 were irregular. All 5 well-defined masses were round/ovoid. Invasion of adjacent soft tissue was found in 5 patients, including 2 patients with bone erosion. According to the shape and margin of the masses, the morphology of tumors could be classified into 3 patterns. The most common pattern was type 2 $(n=16,57.1 \%)$, then type $3(n=7$, $25.0 \%)$ and type $1(n=5,17.9 \%)$. Notably, the shape and size of the lesion in the parotid glands may depend predominantly on the location of the lesion. All masses involving the superficial and deep lobes of the parotid gland were irregular $(n=5)$ or lobulated ( $n=1)$. Additionally, almost half of the masses $(n=5)$ located in the superficial lobe of the parotid gland were round-ovoid, and all masses $(n=11)$ in the superficial lobe of the parotid gland were $<5 \mathrm{~cm}$.

On CT, 23 masses were detected in 23 patients. Compared with the adjacent muscle, lesions showed slight hypoattenuation in 19 patients and isoattenuation in 4 patients. Necrosis was demonstrated in $14.3 \%$ of $(n=4)$ of patients on enhanced CT, and no calcification was found in any patient. After the administration of contrast, lesions showed poor enhancement in 6 patients, moderate enhancement in 13 patients, and intense enhancement in 4 patients. Homogeneous enhancement was found in 14 patients, while heterogeneous enhancement was found in the other 9 patients.

Six patients with 6 masses (parotid glands in 4 patients, submandibular gland in 1, sublingual gland in 1) underwent MR imaging. On T1-weighted imaging, 1 patient had a lesion with a slight hyperintense signal and 5 patients had isointense signal. On 

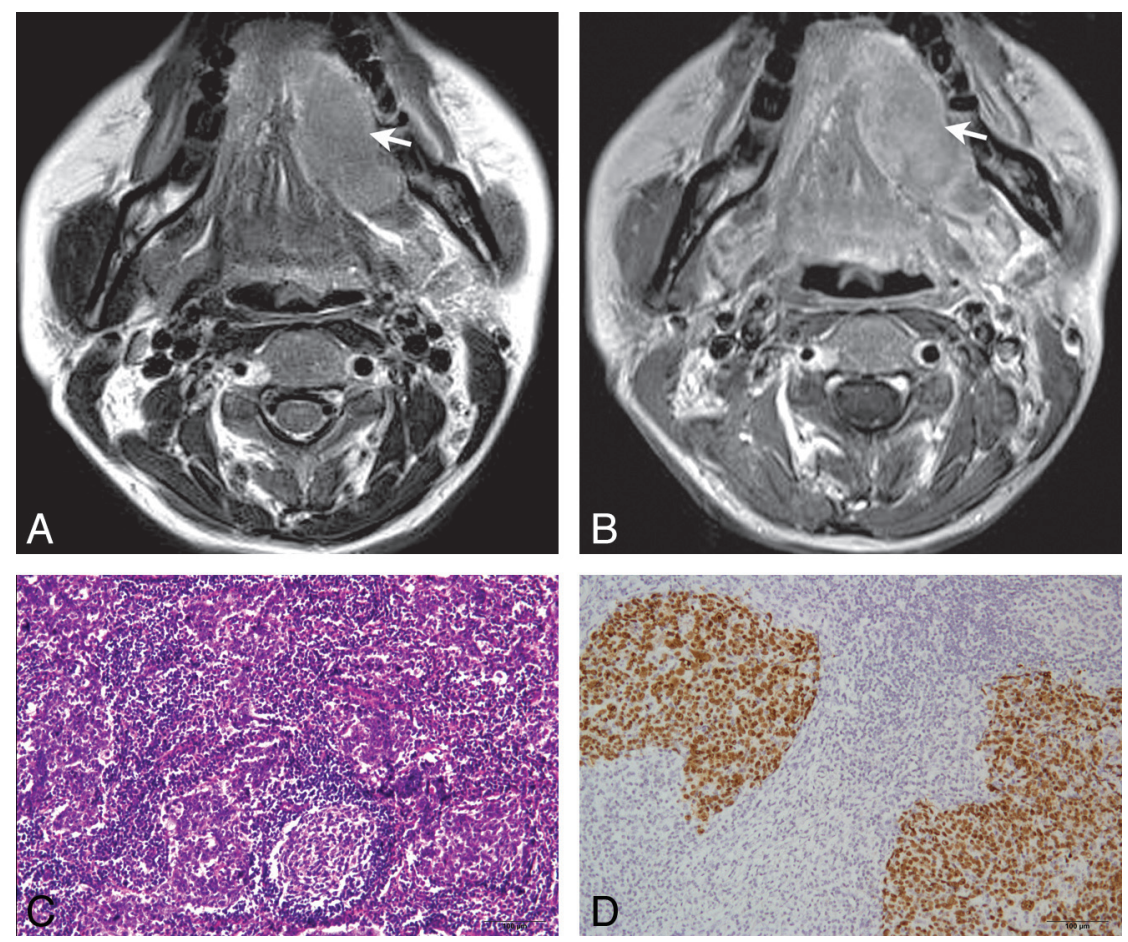

FIG 3. LEC of the left sublingual gland in a 44-year-old woman with a painful mass in the mouth for 8 years. Axial T2-weighted imaging $(A)$ shows a homogeneous slightly hypointensity mass with a partially defined margin in the left sublingual gland (arrow). Axial gadolinium-enhanced T1-weighted image $(B)$ shows the mass with homogeneous moderate enhancement (arrow). Histopathologic examination $(C)$ shows nests of neoplastic epithelial cells separated by abundant lymphoid stroma (hematoxylin-eosin, original magnification $\times 100$ ). In situ hybridization by using a digoxigenin-labeled EBER probe $(D)$ shows the nuclei of the malignant epithelial cells strongly positive but negative in the surrounding lymphocytes (original magnification $\times 100$ ).
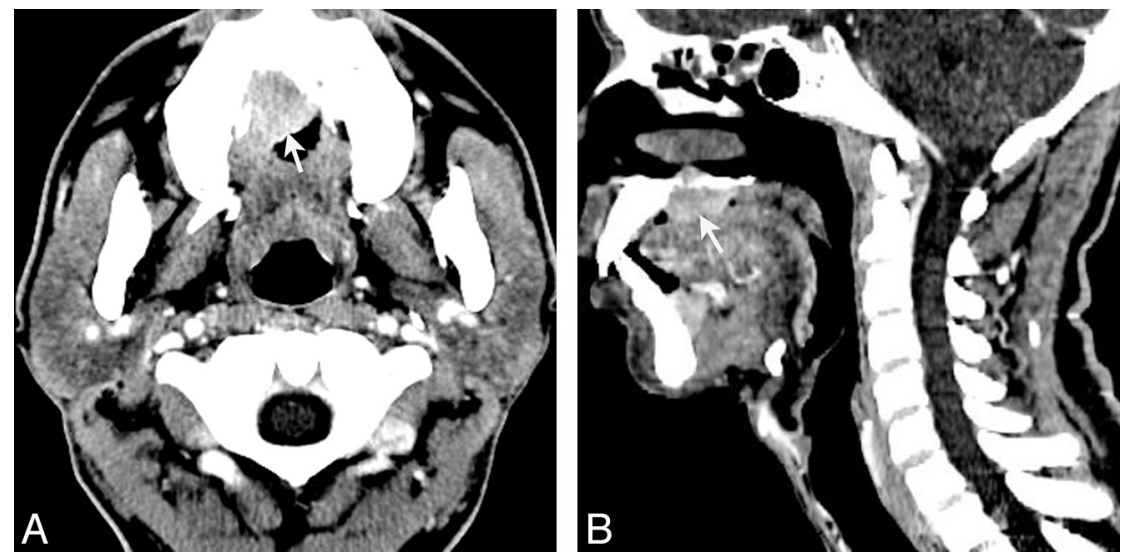

FIG 4. LEC of the right upper palate in a 38-year-old man with a rapidly growing mass in the upper palate for approximately 8 months. Axial contrast-enhanced CT $(A)$ shows the mass with a heterogeneous moderate enhancement (arrow). Sagittal (B) contrast-enhanced CT shows the mass with heterogeneous moderate enhancement (arrow) and bone destruction.

T2-weighted imaging, 4 patients had hypointense signal (Fig $3 A$ ) and 2 patients had slightly hyperintense signal. After the administration of contrast, imaging of 1 patient showed poor enhancement, that of 4 patients showed moderate enhancement, and that in 1 patient showed intense enhancement. Homogeneous enhancement was found in 3 patients, and heterogeneous enhancement was found in 3 patients. Cystic/necrotic areas were not found in any of the 6 patients.

\section{Cervical Lymphadenopathy}

The incidence of neck node involvement was found in $60.7 \%(17 / 28)$ of patients, including 10 patients $(56 \%, 10 / 18)$ with primary lesions in the parotid glands; 6 patients $(75 \%, 6 / 8)$, in the submandibular gland; and 1 patient $(100 \%, 1 / 1)$, in the palate. Cervical lymphadenopathy was unilateral in all patients. The locations of pathologic lymph nodes were consistent with anatomic drainage without skip metastasis. The intraparotid region and level IIb were involved in 8 patients, respectively; level IIa was involved in 7 patients; level Ib was involved in 3 patients; and level III was involved in 1 patient. Necrosis of the lymph nodes was found in 3 patients (Fig 5B).

\section{Histopathologic Results}

The diagnosis of LEC was confirmed histopathologically in all patients by using either surgical resection $(n=27)$ or fineneedle aspiration cytology $(n=1)$. Grossly, all 27 resected tumors were generally grayish or reddish and firm. Calcification was not found in any case, while necrosis was found in 5 cases in these 27 gross specimens. Infiltration into adjacent muscle was found in 3 patients, and bone erosion, in 2 patients. Histopathologic examinations commonly showed infiltrative sheets, nests, and cords of neoplastic epithelial cells separated by lymphoid stroma (Fig 3C). Immunohistochemically, cytokeratin and p63 were positive in 28 and 16 patients, respectively. Twenty-seven tumors showed positive EBER in epithelial neoplastic cells and negative EBER in stromal lymphoid cells and surrounding normal salivary gland tissue (Fig 3D). The remaining tumor showed negative EBER in epithelial neoplastic cells, stromal lymphoid cells, and surrounding normal salivary gland tissue.

\section{DISCUSSION}

LEC of the salivary gland, which has been referred to as undifferentiated carcinoma with lymphoid stroma, malignant lymphoepithelial lesion, malignant lymphoepithelioma, and lymphoepithelial-like carcinoma, ${ }^{1,7}$ is a rare undifferentiated carcinoma with prominent lymphoid stroma and ultrastructural features of squamous cell carcinoma. Increasingly investigators have demonstrated that LEC of the salivary gland is strongly associated with Epstein-Barr virus in endemic areas such as Greenland, Southeast Asia, and Alaska. ${ }^{12}$ In our study, all 28 patients were southern Chinese, and 

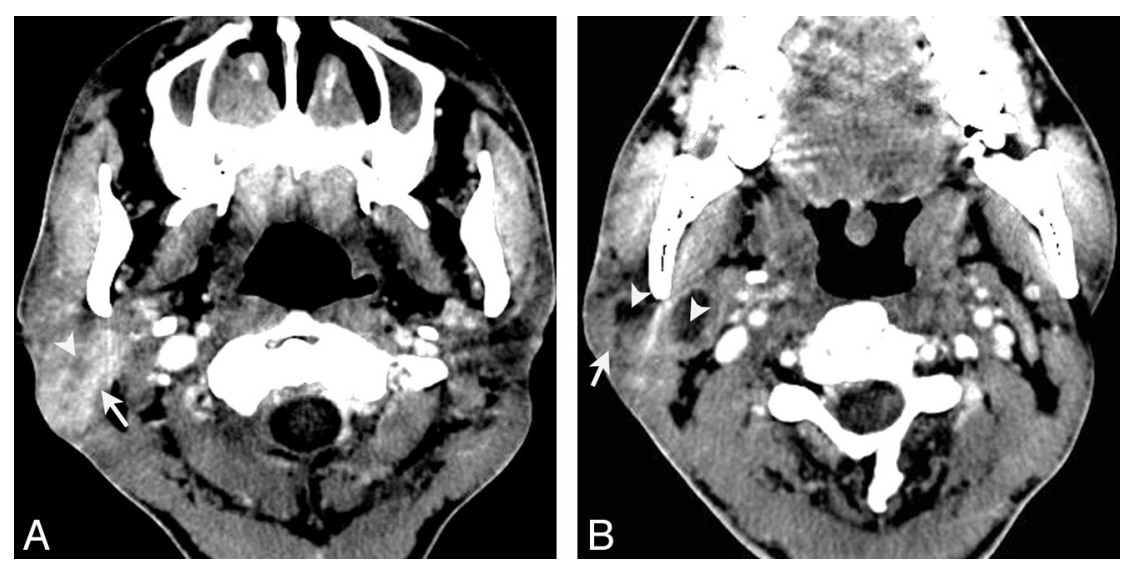

Ahuja et al ${ }^{16}$ reported imaging findings of LECs in minor salivary glands in 4 cases in 1999.

LECs of the salivary glands in our series tended to involve the parotid gland with an ill-defined or partially ill-defined margin. Most LECs in the salivary glands showed slight hypoattenuation on CT, isointense signal on T1WI, and hypointense signal on T2-weighted imaging, with moderate enhancement. Unfortunately, these CT and MR imaging characteristics are nonspecific and do not allow a definitive discrimination from other ma-

FIG 5. LEC of the right parotid gland in a 38-year-old man with painless masses in the right parotid regions for approximately 3 years. Axial contrast-enhanced CT $(A)$ shows an ill-defined mass located in the right parotid gland (arrow) with heterogeneous enhancement and much intratumoral necrosis (arrowhead). Axial contrast-enhanced CT (B) shows multiple enlarged nodes (arrow) in the intraparotid region and level lla, with obvious necrosis (arrowhead).

lignant tumors of the salivary glands. However, most of the lesions had homogeneous attenuation/signal intensity on unenhanced CT and MR imaging; no lesions exhibited cystic degeneration or cal-

96.4\% of tumors were positive for Epstein-Barr virus by using EBER in situ hybridization. Our results support the association of Epstein-Barr virus with salivary gland LEC. In our series, LEC of the salivary gland predominantly occurred in the parotid gland (64.3\%), followed by the submandibular gland (28.5\%). The mean age was 39.3 years, with a male predominance (1.5:1). A slowly growing, palpable mass was present in $71.4 \%$ of patients, and $28.6 \%$ presented with a recent, rapidly growing mass. Almost half of our patients $(42.9 \%)$ experienced a duration of symptoms of $>2$ years, indicating that LECs of the salivary glands had a relatively long duration of symptoms compared with other malignant tumors in the salivary glands, consistent with prior reports. ${ }^{1,2,7}$

Treatment for LEC of the salivary gland includes surgical excision, radiation therapy, and chemotherapy. At present, surgical excision followed by postoperative radiation therapy is considered the treatment of choice for LEC of the salivary gland, and the 5 -year survival rate is reported to range from $50 \%$ to $90 \% .^{2,7}$ The prognosis of salivary gland LEC is reported as better than that in other types of undifferentiated carcinoma of the salivary gland. ${ }^{13}$ The incidence of local recurrence and distant metastasis has varied in previous studies. The reported local recurrence rate ranged from $0 \%$ to $28.9 \% .^{5,7}$ In our cases, 27 patients underwent a surgical excision and 18 patients underwent postoperative radiation therapy. The local recurrence rate in this group was $10.7 \%$. Of our patients, $14.3 \%$ developed distant metastasis to the lung, liver, and brain from 7 to 77 months after diagnosis, comparable with a distant metastasis rate of $2.9 \% \sim 33.3 \%$ in previous studies. ${ }^{5,7,9}$

Fine-needle aspiration cytology is a primary diagnostic tool for salivary gland tumors, ${ }^{14,15}$ but the diagnostic accuracy, sensitivity, and specificity of fine-needle aspiration cytology in the diagnosis of salivary gland lesions are varied owing to diverse morphologic patterns and overlapping features between benign and malignant lesions. In a recent review, the diagnostic accuracy of fine-needle aspiration cytology was $78.6 \%$ in 14 patients with LEC of salivary gland. ${ }^{15}$ Thus, CT and MR imaging are still valuable tools for the diagnosis, preoperative evaluation, and biopsy guidance for patients with LEC. To our knowledge, however, only cification, even in a large mass; and most of our lesions (85.7\%) lacked necrotic regions. These features are, in aggregate, different from those in other common primary malignant tumors of the salivary glands, including mucoepidermoid carcinoma and adenoid cystic carcinoma because these more common malignant tumors typically show heterogeneous attenuation or signal intensity with cystic change and necrosis. ${ }^{17-19}$ Thus, an ill-defined or partially ill-defined mass without cystic regions or calcification and lacking necrosis is typically seen in salivary gland LEC.

In our cases, salivary gland LECs could be classified into 3 morphologic types on the basis of the margin and shape. Morphologic patterns of salivary gland LECs are useful for the differential diagnosis along with other salivary gland tumors. Types 1 (17.9\%) and $3(25.0 \%)$ in our series showed typical morphologic features of benign and malignant tumors in the salivary glands, respectively. Type 2 represented a mass with a lobulated/plaque-like shape and a partially or ill-defined margin. In this study, most tumors belonged to this type. Because most LECs in the salivary glands are less aggressive malignant tumors, ${ }^{7}$ this pattern might be a relatively morphologic characteristic of salivary gland LEC. In addition, previous studies had reported an incidence of lymph node metastases in salivary gland LEC ranging from $10 \%$ to $50 \%{ }^{2,7,20}$ In our study, the incidence of lymph node metastases was up to $60.7 \%$, which was higher than that in previous studies. ${ }^{2,7,20}$ Lymphadenopathy in our cases was unilateral, with the intraparotid region and level IIa the most commonly involved regions. Lymph node metastasis was found to spread along with anatomic drainage. No skip metastasis was present. Lymph node necrosis occurred sometimes, as demonstrated in a previous study. ${ }^{16}$

There are several limitations to our study. First, as a retrospective study, most of our patients underwent CT and only 6 patients underwent MR imaging with conventional $\mathrm{T} 1$ and $\mathrm{T} 2$ sequences. Because MR imaging, particularly MR imaging with diffusionweighted imaging or dynamic contrast-enhanced sequences, is regarded as a better technique for characterizing and delineating salivary gland lesions, ${ }^{21}$ a study using these new techniques may be needed for further determining the imaging features of salivary 
gland LEC. Second, our patients had a follow-up from 5 to 123 months. Four patients had follow-up at $<12$ months, which was less than the time to recurrence or distant metastasis in some other patients. Therefore, it was difficult to conclude the exact long-term recurrence and metastasis rates just on the basis of our data.

\section{CONCLUSIONS}

LEC of the salivary gland displays nonspecific attenuation or signal intensity on CT or MR imaging. It is difficult to make a reliable diagnosis of it on the basis of CT and MR images. However, clinically, it predominantly occurs in Inuit-Yupik and southern Chinese with a strong association with Epstein-Barr virus and a relatively long history. Imaging features, including an intraglandular mass with a lobulated/plaque-like shape and ill- or partially illdefined margins but without calcification and necrosis, accompanied by ipsilateral lymphadenopathy, suggest the diagnosis of salivary gland LEC.

\section{REFERENCES}

1. Schneider M, Rizzardi C. Lymphoepithelial carcinoma of the parotid glands and its relationship with benign lymphoepithelial lesions. Arch Pathol Lab Med 2008;132:278-82

2. Wang CP, Chang YL, Ko JY, et al. Lymphoepithelial carcinoma versus large cell undifferentiated carcinoma of the major salivary glands. Cancer 2004;101:2020-27

3. Hsiung CY, Huang CC, Wang CJ, et al. Lymphoepithelioma-like carcinoma of salivary glands: treatment results and failure patterns. Br J Radiol 2006;79:52-55

4. Hoxworth JM, Hanks DK, Araoz PA, et al. Lymphoepithelioma-like carcinoma of the lung: radiologic features of an uncommon primary pulmonary neoplasm. AJR Am J Roentgenol 2006;186:1294-99

5. Kuo T, Hsueh C. Lymphoepithelioma-like salivary gland carcinoma in Taiwan: a clinicopathological study of nine cases demonstrating a strong association with Epstein-Barr virus. Histopathology $1997 ; 31: 75-82$

6. Hilderman WC, Gordon JS, Large HL Jr, et al. Malignant lymphoepithelial lesion with carcinomatous component apparently arising in parotid gland: a malignant counterpart of benign lymphoepithelial lesion? Cancer 1962;15:606-10

7. Ma H, Lin Y, Wang L, et al. Primary lymphoepithelioma-like carci- noma of salivary gland: 69 cases with long-term follow-up. Head Neck 2013 Aug 22. [Epub ahead of print]

8. Nagao T, Ishida Y, Sugano I, et al. Epstein-Barr virus-associated undifferentiated carcinoma with lymphoid stroma of the salivary gland in Japanese patients: comparison with benign lymphoepithelial lesion. Cancer 1996;78:695-703

9. Leung SY, Chung LP, Yuen ST, et al. Lymphoepithelial carcinoma of the salivary gland: in situ detection of Epstein-Barr virus. J Clin Pathol 1995;48:1022-27

10. Gor DM, Langer JE, Loevner LA. Imaging of cervical lymph nodes in head and neck cancer: the basics. Radiol Clin North Am 2006;44:10110 , viii

11. van den Brekel MW, Stel HV, Castelijns JA, et al. Cervical lymph node metastasis: assessment of radiologic criteria. Radiology 1990;177:379-84

12. Tsai CC, Chen CL, Hsu HC. Expression of Epstein-Barr virus in carcinomas of major salivary glands: a strong association with lymphoepithelioma-like carcinoma. Hum Pathol 1996;27:258-62

13. Larbcharoensub N, Tubtong N, Praneetvatakul V, et al. Epstein-Barr virus associated lymphoepithelial carcinoma of the parotid gland: a clinicopathological report of three cases. J Med Assoc Thai 2006; $89: 1536-41$

14. Singh Nanda KD, Mehta A, Nanda J. Fine-needle aspiration cytology: a reliable tool in the diagnosis of salivary gland lesions. J Oral Pathol Med 2012;41:106-12

15. Colella G, Cannavale R, Flamminio F, et al. Fine-needle aspiration cytology of salivary gland lesions: a systematic review. J Oral Maxillofac Surg 2010;68:2146-53

16. Ahuja AT, Teo PM, To KF, et al. Palatal lymphoepitheliomas and a review of head and neck lymphoepitheliomas. Clin Radiol 1999; 54:289-93

17. Lee YY, Wong KT, King AD, et al. Imaging of salivary gland tumours. Eur J Radiol 2008;66:419-36

18. Thoeny HC. Imaging of salivary gland tumours. Cancer Imaging 2007;7:52-62

19. Christe A, Waldherr C, Hallett R, et al. MR imaging of parotid tumors: typical lesion characteristics in MR imaging improve discrimination between benign and malignant disease. AJNR Am J Neuroradiol 2011;32:1202-07

20. Bosch JD, Kudryk WH, Johnson GH. The malignant lymphoepithelial lesion of the salivary glands. J Otolaryngol 1988;17:187-90

21. Yabuuchi H, Matsuo Y, Kamitani T, et al. Parotid gland tumors: can addition of diffusion-weighted MR imaging to dynamic contrastenhanced MR imaging improve diagnostic accuracy in characterization? Radiology 2008;249:909-16 